\title{
A Brief Overview of the Effects of Melissa officinalis L. Extract on the Function of Various Body Organs
}

\author{
Ali Zarei ${ }^{1}$; Saeed Changizi-Ashtiyani ${ }^{2, *}$; Soheila Taheri ${ }^{3}$; Nasser Hosseini ${ }^{4}$ \\ ${ }_{1}^{1}$ Young Researchers Club, Abadeh Branch, Islamic Azad University, Abadeh, IR Iran \\ ${ }^{2}$ Department of Physiology, Arak University of Medical Sciences, Arak, IR Iran \\ 3 Education Development Center, Arak University of Medical Sciences, Arak, Iran \\ ${ }^{4}$ Department of Medicinal Plants, Arak University, Arak, IR Iran \\ *Corresponding author: Saeed Changizi-Ashtiyani, Department of Physiology, Arak University of Medical Sciences, Arak, IR Iran. E-mail: dr.ashtiyani@arakmu.ac.ir
}

Received: February 18, 2014; Accepted: March 12, 2014

\begin{abstract}
Melissa officinalis (lemon balm) which belongs to the Lamiaceae family is a known herb that has long been used in traditional medicine to treat many disorders, and several studies have been conducted to identify the plant and its healing properties. The purpose of this article is to review a series of studies on the effects of the herb extract on the function of various body organs.

Due to its volatile organic compounds and active constituents such as terpenoids, flavonoids, quercetin, rutin, quercitrin, gallic acid and high antioxidant capacity, the extract of the plant can have a significant role in maintaining health and curing diseases.

In this paper data have been collected from books and scientific papers published in the databases like Science Direct, Web Science, Scopus, EBSCO, Iran medex and PubMed. Search in the interval of the years 2006 to 2012 was carried out. To search for the key words Melissa officinalis, lemon balm, and balm mint were used. About 80 articles was reviewed and after rejection non-related or similar items, in the end about 50 the number was actually cited.

Results indicate that the extract of the plant with its several antioxidant, anti- inflammatory, anti-pain, anti-spasmodic and anti-cancer properties as well as its cholinergic receptor activation have highly significant effects on improving behavioral symptoms, cognitive impairment, insomnia, anxiety, and stress.

However, these studies have been vast and sparse, and doing more focused and extensive researches in this area is recommended.
\end{abstract}

Keywords: Lipids; Liver; Antioxidants

\section{Context}

Medicinal plants have had a great role in providing health and treatment as well as disease prevention in human communities $[1,2]$. Medicinal herbs are very rich in secondary metabolites which have profound physiological effects on the function of mammalian tissues in health and disease conditions [3, 4]. Melissa officinalis (lemon balm) is one of these known herbs that has been used since a very long time ago for the treatment of many illnesses like headaches, gastrointestinal diseases, neurological diseases and rheumatoid [5, 6]. M. officinalis from Lamiaceae family (Figure 1), with other common names like bee balm, garden balm, melissa, melissengeist, is a perennial herbaceous plant which grows vastly from the central and southern Europe to Iran and central Asia. It is also cultivated worldwide for its edible properties [7-9]. This herb has been used extensively in traditional medicine and the history of it goes back to more than 2,000 years ago. The plant has been used in a variety of ways from a sedative and mild hypnotic drug [8-10], and reducing the heart rate, antibacterial, antiinflammatory, anti- virus, antispasmodic, antioxidant, to a neurotherapeutic agent, peripheral analgesic, as well as a binding agent to cholinergic receptors [7-11].

\section{Evidence Acquisition}

Avicenna, the Iranian eminent physician and philosopher introduced this plant as vitality tonic and as a sedative drug to treat neurological disorders [11].

The most important ingredients in the plant are known to be phenolic compounds, rosmarinic acid, caffeic acid, cholinergic acid, metrilic acid; flavonoids such as luteolin, apigenin and monoterpene derivatives; the sesquiterpenes including beta-caryophyllene and germacrene; triterpenes such as oleanolic and ursolic acid; volatile oil, and tannins [8]. The aim of present article is to give a brief overview of the health benefits of M. officinalis extract and is concentrated on the functions of liver, thyroid, cancer, immune system, adipose tissue and also plasma concentrations of some biochemical factors.

Copyright (C) 2015, Zahedan University of Medical Sciences. This is an open-access article distributed under the terms of the Creative Commons Attribution-NonCommercial 4.0 International License (http://creativecommons.org/licenses/by-nc/4.0/) which permits copy and redistribute the material just in noncommercial usages, provided the original work is properly cited. 

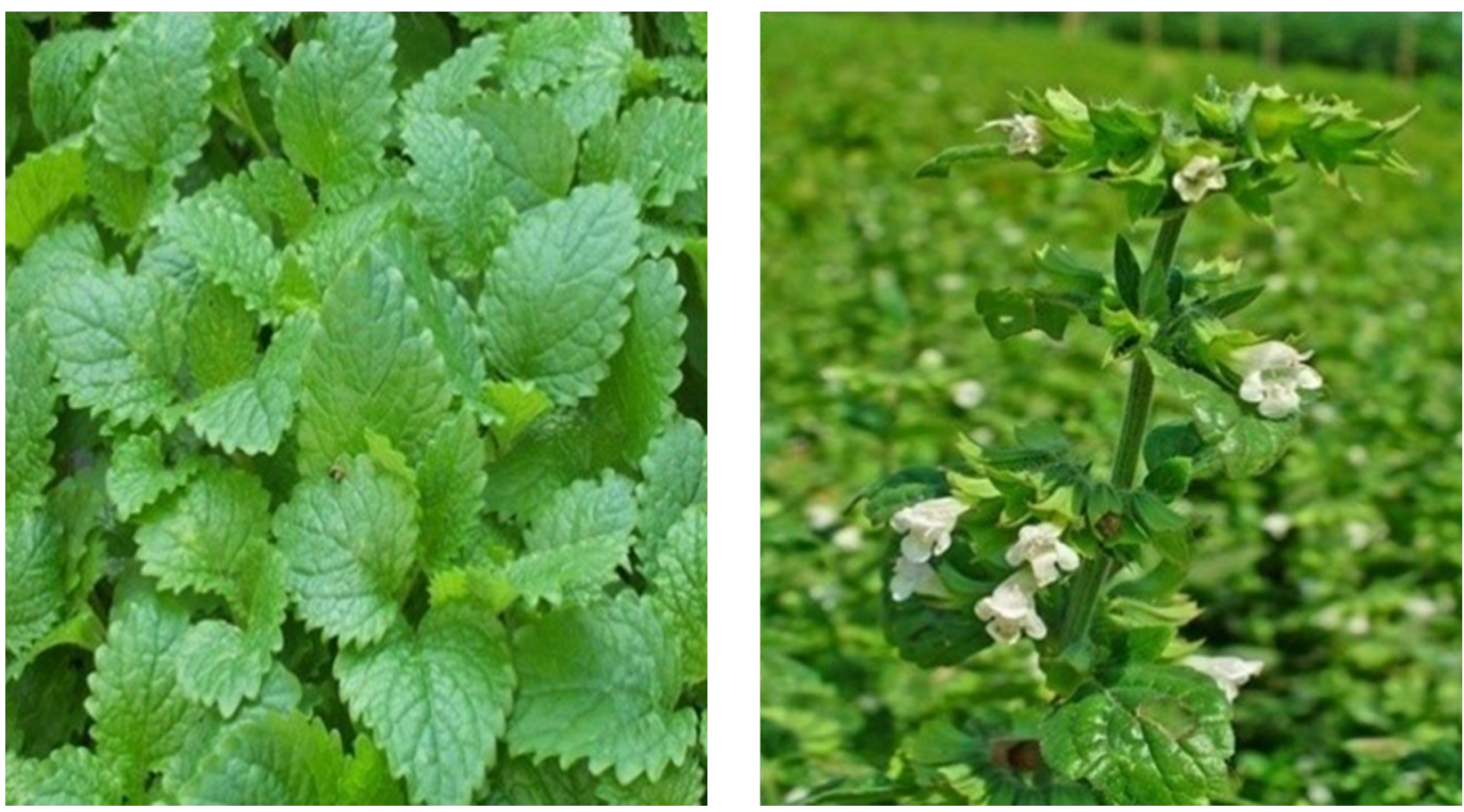

Figure 1. Aerial Parts of Melissa officinalis

This review article is a survey of the empirical papers of several authors who have examined the role of $M$. officinalis extract in different body tissues. To conduct a literature review, the authentic english sources in different databases like Science Direct, PubMed, Google Scholar, and Persian databases including: Scientific Information Database (SID), Iran Articles Database of Medical Sciences, Iran medex, Iranian Research Institute for Information Science and Technology (Iran Doc), the publications database (Magiran) and library archives, as well as articles published in scientific journals, Persian and English, have been used. Search in the interval of the years 2006 to 2012 was carried out. To search for the key words M. officinalis, lemon balm, and balm mint were used. About 80 articles was reviewed and after rejection non-related or similar items, in the end about 50 the number was actually cited.

\section{Results}

The effect of M. officinalis extract on lipid profiles: Hypertriglyceridemia is one of the most important factors in the development of cardiovascular diseases [5]. Among effective pharmacological agents in treating these diseases, we can refer to statins, clofibrates and niacin. However, in general these compounds are not effective in reducing cholesterol concentration and are often associated with adverse effects and drug toleration, [12-19]. Therefore, all efforts have been concentrated on prevention strategies like phytochemical diet.

According to recent studies, M. officinalis essential oil has significant metabolic effects in vivo. Terpenoids are among the essential compounds in herbal oils that in- duce hypolipidemic effects by inhibiting liver biosynthesis and formation of cholesterol nucleus in bile. Studies have shown that the essential oil compounds of Plantago asiatica inhibit mRNA and protein expression of HMGCOA reductase in HePG2 cells and C57BL/6 cells in rats [19].

Although the lipid-lowering mechanism of M. officinalis is not clearly known, studies have shown that regular and daily drinking of $M$. officinalis tea may improve the metabolic parameters like cholesterol and triglycerides in humans [5]. In addition, M. officinalis has the potential to inhibit hypercholesterolemia, to reduce serum lipid concentrations and lipid peroxidation in the liver of hypercholesterolemic rats [20]. Evidence show that herbal oils, including M. officinalis oils have various pharmacological effects which are mainly related to volatile terpenoids such as geranial, cineol and caffeic acid [5].

In a study on rats with hypercholesterolaemia, Changizi-Ashtiyani et al. have shown that M. officinalis and barberry extract can reduce serum cholesterol, low-density lipoprotein (LDL) and triglyceride. However, the hypolipidemic properties of alcoholic extract of $M$. officinalis are more than those of barberry root which are most likely related to the antioxidant properties of $M$. officinalis and its effect on increasing thyroid hormone [21].

Jun et al. in a study on the impact of $M$. officinalis extract on reducing plasma triglyceride levels has shown that this is due to the presence of quercetin compounds in the plant and its possible effect on the inhibition of lipid peroxidation [5].

M. officinalis essential oil also has anti diabetic properties and improves glucose tolerance and adjusts the ex- 
Zarei A et al.

pression of the genes involved in hepatic gluconeogenesis studies by Chung et al. have shown that daily uptake of $M$. officinalis at low doses can cause hypoglycemia by increasing glucose uptake and its metabolism in the liver, as well as by gluconeogenesis inhibition [22].

Study of Zarei et al. on the effect of the M. officinalis extract on the activity of liver enzymes in hypercholesterolemic rats showed that the enzymes level in hypercholesterolemic group receiving the extract reduced [23]. Various reasons have been proposed for the increase in liver enzymes in hypercholesterolemic rats. For example one theory says that the accumulation of lipids in the liver raises a pathological state causing fibrous changes and eventually cirrhosis and hepatic dysfunction. Following these disorders, levels of liver enzymes, particularly ALT increases [24]. On the other hand, hyperlipidemia will also stimulate the production of free radicals [25].

The impact of $M$. officinalis extract on reducing liver enzymes is known to be due to its powerful antioxidant properties. This plant contains phenolic compounds, which are among the most important antioxidant agents.

These compounds, especially flavonoids have a protective effect on liver against damages caused by free radicals because of their inhibitory effect on the cytochrome system. Flavonoids can also protect the cells against glutathione depletion by increasing the capacity of the antioxidant enzymes (glutathione reductase, oxidase and catalase) [23].

In another study, Bolkent et al. examined the effect of $M$. officinalis extract on hyperlipidemic rats' liver in which liver cholesterol, total lipid, lipid peroxidation, and liver enzymes reduced while the glutathione levels increased [20]. The effect of M. officinalis extract on the function of pituitary-thyroid axis: The results of the study done by Zarei et al. on the impact of M. officinalis extract on the function of pituitary-thyroid axis in rats with hypercholesterolemia showed increased thyroid hormone levels and reduced levels of thyroid stimulating hormone (TSH) [26]. Initially M. officinalis extract increases the secretion of TRH and TSH and consequently it increases the amount of T3 and $\mathrm{T} 4$, this increase in T3 and T4 can finally reduce TSH level through exerting a negative feedback effect. Studies show that some neuromodulators and neurotransmitters control the neurons which secret TRH hormone in the hypothalamus. Some of these neurotransmitters, such as catecholamines (epinephrine, norepinepherine, serotonin and dopamine) have an increasing role and some, such as interleukin-1 (IL1) and gamma aminobutyric acid (GABA) have a decreasing role [26, 27].

On one hand, the flavonoid compounds in the plant preserve and conserve catecholamines by inhibiting the monoamine oxidase enzyme (MAO) and on the other hand, by inhibiting the cyclooxygenase enzyme, they reduce the production of prostaglandins and inflammatory cytokines in response to inflammatory stimuli. In this way they can also exert their anti-inflammatory effects [28]. M. officinalis extract has two mechanisms on
GABA: one is its GABAergic property to inhibit GABA and the other one is its cholinergic property which inhibits acetylcholinesterase, and enhances the capabilities of brain cholinergic receptors $[26,29]$.

According to studies, GABA inhibits and reduces TRH secretion. So, if GABA is inhibited, TRH will increase and subsequently the secretion of thyroid hormones will increase too. Studies also indicate that the relation between fat and leptin, is direct, but fats and thyroid hormone levels have an inverse and significant relation. Since M. officinalis extract can reduce blood lipid levels, it is most likely that at least part of this effect is exerted by increasing thyroid hormone [21].

Antioxidant effects of M. officinalis: M. officinalis has powerful antioxidant effects and these effects probably are exerted through the rosmarinic acid and the benzodioxole present in the extract. The antioxidant effects of these compounds are up to ten times stronger than the effects of those of vitamins B and C. In this way, M. officinalis, like vitamin C, can moderate the neurotoxic effects of chemical drugs $[7,30]$.

In addition, compounds such as acid linoleic acid, carnosic acid, urosolicacidare are also present in the extract all of which have antioxidant properties. M. officinalis compounds are able to bind to acetylcholine; moreover, they contain an inhibitory effect on the acetylcholinesterase (AChE) enzyme and thus are able to improve cognitive functions like memory [31].

Pereira et al. has also shown that the antioxidant activity of phenolic compounds in the plant extract is mostly because of rutin, quercitrin, garlic acid, and quercetin. The highest antioxidant properties of compounds belong to quercetin and then to garlic acid, quercitrin and rutin respectively. In this study it has been shown that M. officinalis extract has a protective function against the oxidative damage caused by different peroxidative agents. These agents can cause lipid peroxidation in a number of ways. Therefore, this herbal extract can inhibit the production of chemically active species in their early stages, or later, it may block lipid peroxidation through various processes. Finally, the researchers state that M. officinalis extract can prevent neurological diseases associated with oxidative stress [32].

In another study, Martins et al. have tested the effect of the M. officinalis extract on oxidative stress induced by manganese (Mn). Manganese is an essential element for biological systems, but its increased level may lead to neurodegenerative diseases. Although the mechanism of this neurotoxicity is not fully clear, the oxidative stress has a key role in the development of these diseases. In this study, it has been shown that that manganese increases the level of thiobarbituric acid reactive substances (TBARS) as a marker of oxidative stress in hippocampus and the striatum. In that study the amount of the marker in animals treated with the extract had decreased, too [33].

Analgesic and anti-inflammatory effects of Melissa of- 
ficinalis: Using herbs as pain relief has a long history in the world of medicine. One of these plants is M. officinalis whose analgesic effect is not dose-dependent. The analgesic effect is likely done by opioid receptors. Acute analgesic effect of this extract does not differ much from that of morphine and aspirin. However, its chronic analgesic properties are less than morphine and aspirin. So, the peripheral analgesic function of the extract seems to be less considerable than the central analgesic mechanism [34].

The anti-nociceptive and anti-inflammatory effects of M. officinalis are attributed to the rosmarinic acid and flavonoids and terpenoids present in the extract. Probably flavonoids have a more effective role by facilitating prostaglandin synthesis. The analgesic activity of flavonoids is through moderating opioidergic mechanism $[34,35]$

Drozd and Anuszewska have examined the effect of $M$. officinalis extract on the immune system response in rats. They have found that this effect is comparable to the effect of levamisole, which is known for its effect on the immune system. Aqueous extract of M. officinalis is effective on both blood and cellular responses [36].

M. officinalis oil extract derived from the leaves of the plant contains nerol (30.44\%), citral (27.03\%), isopolcule (22.02\%), cariophiline $(2.29 \%)$, oxide carolyn $(1.24 \%)$ and citronella (1.06\%). In animal models its clear and strong anti-inflammatory and analgesic effects has been shown in comparison to those of a standard analgesic and antiinflammatory drug (indomethacin) [37]. A phytochemical study on M. officinalis suggests that among its phenolic compounds, rosmarinic acid and galic acid have the highest and the lowest concentrations respectively [38].

Anxiolytic effect of M. officinalis extract: Anxiety disorders are the most common mental disorders with the prevalence of 10 - 30 percent. Results of several studies indicate that the aqueous extract of M. officinalis with the dose of $5 \mathrm{mg} / \mathrm{kg}$ has anxiolytic effect, whereas at higher doses it has a sedative effect in rats. So, the anxiolytic effects are dose dependent and may be applied through opioid receptors [39].

In another study conducted to examine the effect of traditional herbals on neurological disorders, including Alzheimer's, epilepsy and depression, it is concluded that the $M$. officinalis extract does not have any role in the serotonin transport; however, it is involved in AChE activity. Moreover, $M$. officinalis extract has a moderate affinity to gamma-aminobutyric acid receptor (GABA). So it seems that the anxiolytic properties of $M$. officinalis may be due to binding to GABA type receptors [40].

In some European countries, the herb extract is used as pain reliever and relaxant especially when there is disruption in the first stage of sleep by some unpleasant stressful factors [41]. In another study on the effect of the extract on people with Alzheimer's, it is found that M. officinalis extract can reduce agitation in Alzheimer's patients. The administration of the citronella, taken from the extract, has caused sound sleep and has reduced muscle tone in people with sleep and neurological dis- orders. M. officinalis extract has also resulted in a significant improvement in insomnia, irritability, headaches, and heart disease in mentally ill patients [39]. Ibarra et al. study on the effects of chronic administration of $M$. officinalis extract on anxiety reactions and circadian activities has shown that the use of this extract reduces anxiety like reactivities. Because the herb extract contains significant amounts of rosmarinic acid, oleanolic acid, ursolicacid, and triterpenoids, it is most likely that these compounds inhibit GABA transport activity and increase GABA level in brain $[42,43]$.

Akhondzadeh et al. study in which M. officinalis extract was administrated to patients with mild to moderate Alzheimer's for 4 months has shown a significant improvement in their behavioral and cognitive symptoms and a decrease in anxiety and apprehension. The incidence of these effects is likely due to the stimulating function of acetylcholine receptors present in the plant [44]. The effect of the extract on the nervous system: Cerebral ischemia by causing metabolic disorders leads to neuronal death. Researchers in a study on the effect of M. officinalis on death inducing hypoxia, in cultured cortical neurons in vitro and in the ischemic hippocampus in vivo (rat hippocampus) have shown that using the oil extract significantly protects neurons in hypoxia culture. $M$. officinalis extract significantly decreases the activity of caspase 3 and of TUNEL-positive cells (cells that are located in the CA1 region of the hippocampus). M. officinalis also inhibits the production of malondialdehyde (MDA), and reduces antioxidant capacity in hippocampus. mRNA levels of proinflammatory cytokines, TNFX and IL1B and

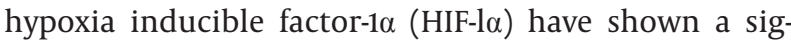
nificant increase after ischemia. The administration of M. officinalis extract inhibits the expression of HIF-la. Studies have also shown that $M$. officinalis extract can be used as a protective agent in several neurologic disorders associated with cerebral ischemia [45].

Hassanzadeh et al. study suggests that the protective effect of $M$. officinalis plant against apoptosis is resulted from the impact of methylenedioxy-methylamphetamine (MDMA) on nerve cells, part of which is probably because of the removal of free radicals and amino oxidase inhibitors. The in vitro study they conducted on neuronal cells showed that daily use of $M$. officinalis extracts within 3 weeks led to cell proliferation, neuroblast differentiation and integration and increase of GABA [46].

M. officinalis effect on Alzheimer's disease, seizures and epilepsy: Alzheimer's disease is a degenerative neurological disease which is believed to cause dementia. Dementia is characterized by a progressive loss of cognitive powers, which leads to social or occupational disability [47]. M. officinalis has sedative and relaxing effects and in its homogenate notably binds to brain from nicotinic and muscarinic acetylcholine receptors and also inhibits the effects of acetylcholine esterase enzyme. Thus, by regulating the cholinergic system it is helpful in Alzheimer's treatment and adjusting mood and cog- 
Zarei A et al.

nitive processes [7].

Epilepsy includes a group of disorders caused by abnormal electrical activity in the brain. Epileptic attacks may appear as seizure, convulsion, or other nervous disorders (in sensory, cognitive, and affective functions). The ingredients in M. officinalis plant imitate nicotine effect in the body and accelerate starting time and increases the duration of the attack. M. officinalis plant is effective as a pretreatment in modulating the seizure symptoms caused by the injection of pentylenetetrazol (PTZ) in rats [47].

Anti-tumor effects of M. officinalis extract: Saraydin et al. studied the effect of $M$. officinalis extract on cytotoxicity of breast cancer cell lines (MCF-7, MDA-468, and MDAMBA-231). The results showed that the extract contained active cytotoxicity against all 3 cancer cell lines. Protein expression of caspase-7 and (TUNEL) positive cells in a group of rats treated with the extract was much more than those of the control group, while Ki-67 expression had reduced. In addition, in vitro studies indicated that inhibition of tumor volume in the group treated with the extract group compared to the control group of rats reduced by $40 \%$. Finally, we can say that $M$. officinalis extracts has an anti tumor potential against breast cancer [48]. de Carvalho et al. also showed that the M. officinalis extract had anti mutagenic or antigeotaxis properties [49].

\section{Conclusions}

M. officinalis plant is one of the oldest and most known herbaceous aromatic plants, and has been used in different forms such as oil extracts, aqueous extract, and applying ointment and compress. There are a variety of active ingredients in the plant which make its antioxidant, sedative, neuroprotective, anti-anxiety and hypnotic properties possible. Its metabolic interventions occur when the plant extract acts to protect the liver and reduce the amount of lipid profiles and influence the thyroid hormone function. The presence of active and effective antioxidants, especial ability to inhibit the production of free radicals, as well as cytotoxic and anti-mutagenic effects, have given a unique feature to the plant. It seems that this ancient medicinal herb has still enormous potential that lies ahead of keen researchers to conduct a lot of biological research.

\section{Acknowledgements}

The present article summarized and updated the latest finding about Melissa officinalis. This was done as part of the research project No: 90-123-12 in Arak University of Medical Sciences. We would like to appreciate the kind help we received from research deputy of the university.

\section{Conflict of Interest}

The authors declare no conflict of interest.

\section{Funding/Support}

Arak University of Medical Sciences.

\section{References}

1. Changizi Ashtiyani S, Najafi H, Jalalvandi S, Hosseinei F. Protective effects of Rosa canina L fruit extracts on renal disturbances induced by reperfusion injury in rats. Iran J Kidney Dis. 2013;7(4):290-8.

2. Changizi Ashtiyani S, Zohrabi M, Hassanpoor A, Hosseini N, Hajihashemi S. Oral administration of the aqueous extract of Rosmarinus officinalis in rats before renal reperfusion injury. Iran J Kidney Dis. 2013;7(5):367-75.

3. Taheri S, Zarei A, Changizi Ashtiyani S, Rezaei A, Zaheiri S. Evaluation of the effects of hydroalcoholic extract of Berberis vulgaris root on the activity of liver enzymes in male hypercholesterolemic rats. Avicenna J Phytomed. 2012;2(3):153-61.

4. Zarei A, Shariati M, Shekar Forosh S, Ashtiyani S, Rasekh F. The effect of Physalis alkekengi extract on the physiologic function of organ tissues: a mini-review [In Persian]. Arak Med Univ J. 2012;15(7):94-104.

5. Jun HJ, Lee JH, Jia Y, Hoang MH, Byun H, Kim KH, et al. Melissa officinalis essential oil reduces plasma triglycerides in human apolipoprotein E2 transgenic mice by inhibiting sterol regulatory element-binding protein-1c-dependent fatty acid synthesis. J Nutr. 2012;142(3):432-40.

6. Wichtl M. Herbal drugs and phytopharmaceuticals: a handbook for practice on a scientific basis. Medpharm GmbH Scientific Publishers; Germany: Medpharm Press;2004.

7. Behnam Rassouli M, Ghayour N, Afsharian M, Tehranipour M Ghayour MB. The protective effects of Melissa officinalis leaves usage on learning disorder induced by lead acetate administration during pre and postnatal periods in rats [In Persian]. Arak Med Univ J. 2010;13(1):97-104.

8. Rasmussen P. Lemon balm--Melissa officinalis; also known as lemon balm, bee balm, garden balm, Melissa, melissengeist. $J$ Prim Health Care. 2011;3(2):165-6.

9. Chen XK, Yang Q, Smith G, Krewski D, Walker M, Wen SW. Environmental lead level and pregnancy-induced hypertension. Environ Res. 2006;100(3):424-30.

10. NourEddine D, Miloud S, Abdelkader A. Effect of lead exposure on dopaminergic transmission in the rat brain. Toxicology. 2005;207(3):363-8.

11. Naghibi F, Mosaddegh M, Mohammadi Motamed M, Ghorbani A. Labiatae family in folk medicine in Iran: from ethnobotany to pharmacology [In Persian]. Iran J Pharm Res. 2005; 4(2): 63-79.

12. Mahmoodi M. Experimental study to evolution the pretreatment of Melissa officinalis extract against by phenyltetrazol in Wistar rats. [In Persian]. J Kerman Univ Med Sci. 2001;8(2):88-94.

13. Shekar-Foroosh S, Changizi-Ashtiyani S, Akbarpour B, Attari MM Zarei A, Ramazani M. The effect of alcoholic extract of physalis alkekengi on serum concentration of thyroid hormones in rats. Zahedan J Res Med Sci. 2012;14(5):7-11.

14. Stohler R, Keller U, Riesen WF. Effects of simvastatin and fenofibrate on serum lipoproteins and apolipoproteins in primary hypercholesterolaemia. Eur J Clin Pharmacol. 1989;37(2):199-203.

15. Drexel H. Statins, fibrates, nicotinic acid, cholesterol absorption inhibitors, anion-exchange resins, omega-3 fatty acids: which drugs for which patients? Fundam Clin Pharmacol. 2009;23(6):687-92.

16. Blane GF. Comparative toxicity and safety profile of fenofibrate and other fibric acid derivatives. Am J Med. 1987;83(5):26-36.

17. Morgan JM, Capuzzi DM, Guyton JR. A new extended-release niacin (niaspan): efficacy, tolerability, and safety in hypercholesterolemic patients. Am J Cardiol. 1998;82(12A):29U-34U.

18. Verges B. Fenofibrate therapy and cardiovascular protection in diabetes: recommendations after FIELD. Curr Opin Lipidol. 2006;17(6):653-8.

19. Chung MJ, Park KW, Kim KH, Kim CT, Baek JP, Bang KH, et al. Asian plantain (Plantago asiatica) essential oils suppress 3-hydroxy3-methyl-glutaryl-co-enzyme A reductase expression in vitro and in vivo and show hypocholesterolaemic properties in mice. $\mathrm{Br} J$ 
Nutr. 2008;99(1):67-75.

20. Bolkent S, Yanardag R, Karabulut-Bulan O, Yesilyaprak B. Protective role of Melissa officinalis L. extract on liver of hyperlipidemic rats: a morphological and biochemical study. J Ethnopharmacol.2005;99(3):391-8.

21. Changizi Ashtiyani S, Zarei A, Taheri S, Rezaei A, Golshan M, Ghafarzadegan R. A comparative study of hypolipidemic activities of the extracts of Melissa officinalis and Berberis vulgaris in rats. $J$ Med Plants. 2013;3(47):38-47.

22. Chung MJ, Cho SY, Bhuiyan MJ, Kim KH, Lee SJ. Anti-diabetic effects of lemon balm ( Melissa officinalis) essential oil on glucose- and lipid-regulating enzymes in type 2 diabetic mice. $\mathrm{Br} J$ Nutr. 2010;104(2):180-8.

23. Zarei A, Changizi Ashtiyani S, Taheri S, Rasekh F. Comparison between effects of different doses of Melissa officinalis and atorvastatin on the activity of liver enzymes in hypercholesterolemia rats. Avicenna J Phytomed. 2014;4(1):15-23.

24. Stolk L, Perry JR, Chasman DI, He C, Mangino M, Sulem P, et al. Meta-analyses identify 13 loci associated with age at menopause and highlight DNA repair and immune pathways. Nat Genet. 2012;44(3):260-8.

25. Nazari A, Delfan B, Shahsavari GH. The effect of Satureja Khuzestanica on triglyceride, glucose, creatinine and alkaline phosphatase activity in rat. Shahrekord Univ Med Sci. 2005;7(2).

26. Zarei A, Changizi Ashtiyani S, Sokhandani M, Rezaei A, Zaheiri S, Taheri S. Comparison between the effects of the alcholic extract of Mellissia officinalis and atorvastatin on serum levels of thyroid hormones in hypercholesterolemic male rats [In Persian]. Zahedan J Res Med Sci. 2013;15(8):6-12.

27. Gharib-Naseri MK, Handali S, Hoseini H. Antispasmodic activity of Physalis alkekengi L. leaf hydroalcoholic extract on rat ileum. Iran J Med Arom Plants. 2007;23:340-9.

28. Vaez G, Tavasoli Z, Ranjbar-Bahadori S. Study on the different dosages of Elaeagnus angustifolia aqueous extract with and without morphine on the antinociceptive rate in mice [In Persian] Pejouhesh. 2011;35(1):27-33.

29. Hossini E, Sadeghi H, Daneshi A. Evaluation of hydroalcoholic extract of peganum harmala on pituitary-thyroid hormones in adult Male rats [In Persian]. Armaghane-danesh. 2010;14(4):23-30.

30. Dastmalchi K, Damien Dorman HJ, Oinonen PP, Darwis Y, Laakso I, Hiltunen R. Chemical composition and in vitro antioxidative activity of a lemon balm (Melissa officinalis L.) extract. LWT-Food Sci Technol. 2008;41(3):391-400.

31. Rostami S., Momeni Z, Behnam R, Ghayour N. Comparison of antioxidant effect of Melissa officinalis leaf and vitamin C in lead acetate induced learning deficits in rat [In Persian]. Sci Res J Shahed Univ. 2010;17(86):47-54.

32. Pereira RP, Fachinetto R, de Souza Prestes A, Puntel RL, Santos da Silva GN, Heinzmann BM, et al. Antioxidant effects of different extracts from Melissa officinalis, Matricaria recutita and Cymbopogon citratus [In Persian]. Neurochem Res. 2009;34(5):973-83.

33. Martins EN, Pessano NT, Leal L, Roos DH, Folmer V, Puntel GO, et al. Protective effect of Melissa officinalis aqueous extract against Mn-induced oxidative stress in chronically exposed mice. Brain Res Bull. 2012;87(1):74-9.

34. Miladi Gorgi H, Vafaee AA, RashidiPoor A, Taherian AA, Jarrahi M,
Emami Abarghoee M, et al. The role of opioid receptors on anxiolytic effects of the aqueous extract of Melissa officinalis in mice. Razi J Med Sci. 2005;12(47):145-53.

35. Anjaneyulu M, Chopra K. Quercetin, a bioflavonoid, attenuates thermal hyperalgesia in a mouse model of diabetic neuropathic pain. Progress Neuro Psychopharmacol Biol Psychiatr 2003;27(6):1001-5.

36. Drozd J,Anuszewska E. The effect of the Melissa officinalis extract on immune response in mice. Acta Pol Pharm. 2003;60(6):467-70.

37. Bounihi A, Hajjaj G, Alnamer R, Cherrah Y, Zellou A. In vivo potential anti-inflammatory activity of Melissa officinalis L. essential oil. Adv Pharmacol Sci. 2013:101759.

38. Arceusz A, Wesolowski M. Quality consistency evaluation of Melissa officinalis L. commercial herbs by HPLC fingerprint and quantitation of selected phenolic acids. J Pharm Biomed Anal. 2013;83:215-20.

39. Ulbricht C, Brendler T, Gruenwald J, et al. Lemon balm (Melissa officinalis $\mathrm{L}$ ): An evidence-based systematic review by the natural standard research collaboration. J Herbal Pharmacotherapy. 2005; 5(4): 71-114.

40. Salah SM, Jager AK. Screening of traditionally used Lebanese herbs for neurological activities. J Ethnopharmacol. 2005;97(1):145-9.

41. Soulimani R, Fleurentin J, Mortier F, Misslin R, Derrieu G, Pelt JM Neurotropic action of the hydroalcoholic extract of Melissa officinalis in the mouse. Planta Med.1991;57(2):105-9.

42. Ibarra A, Feuillere N, Roller M, Lesburgere E, Beracochea D. Effects of chronic administration of Melissa officinalis L. extract on anxiety-like reactivity and on circadian and exploratory activities in mice. Phytomedicine. 2010;17(6):397-403.

43. Awad R, Levac D, Cybulska P, Merali Z, Trudeau VL, Arnason JT. Effects of traditionally used anxiolytic botanicals on enzymes of the gamma-aminobutyric acid (GABA) system. Can J Physiol Pharmacol. 2007;85(9):933-42.

44. Akhondzadeh S, Noroozian M, Mohammadi M, Ohadinia S, Jamshidi AH, Khani M. Melissa officinalis extract in the treatment of patients with mild to moderate Alzheimer's disease: a double blind, randomised, placebo controlled trial. J Neurol Neurosurg Psychiatry. 2003;74(7):863-6.

45. Bayat M, Azami Tameh A, Hossein Ghahremani M, Akbari M, Mehr SE, Khanavi M, et al. Neuroprotective properties of Melissa officinalis after hypoxic-ischemic injury both in vitro and in vivo. Daru. 2012;20(1):42.

46. Hassanzadeh G, Pasbakhsh P, Akbari M, Shokri S, Ghahremani M, Amin G, et al. Neuroprotective properties of melissa officinalis L. extract against ecstasy-induced neurotoxicity. Cell J. 2011;13(1):25-30.

47. Bhat JU, Nizami Q, Aslam M, Asiaf A, Ahmad S, Ahmad S. Antiepileptic activity of the whole plant extract of Melissa officinalis in Swiss albino mice. Int J Pharm Sci Res. 2012;3(3):886-9.

48. Saraydin SU, Tuncer E, Tepe B, Karadayi S, Ozer H, Sen M, et al. Antitumoral effects of Melissa officinalis on breast cancer in vitro and in vivo. Asian Pac J Cancer Prev. 2012;13(6):2765-70.

49. de Carvalho NC, Correa-Angeloni MJ, Leffa DD, Moreira J, Nicolau $\mathrm{V}$, de Aguiar Amaral P, et al. Evaluation of the genotoxic and antigenotoxic potential of Melissa officinalis in mice. Genet Mol Biol. 2011;34(2):290-7. 